\title{
HOMOTOPY INVERSES FOR NERVE
}

\author{
BY RUDOLF FRITSCH AND DANA MAY LATCH
}

Whitehead [19] introduced the category of $C W$ complexes as the appropriate category in which to do homotopy theory. Eilenberg, Mac Lane and Zilber ([1], [2]) defined the notion of simplicial set in the early 50's and Kan $([5],[6],[7])$ introduced the necessary conditions to do homotopy theory in this category. The equivalence of these categories under adjoint functors (see [14] , [6], [5], [4]) played an important role in the development of geometric topology. In the late 60's, Quillen [16] used the notion of classifying space for a small category [17], and showed the importance of doing homotopy theory in the category of small categories. Latch [8] recently showed that the category of small categories and the category of simplicial sets were equivalent "up to homotopy," but not by using adjoint functors . In this paper, adjoint pairs are given and a general criteria for such adjoint functors to induce a "homotopy equivalence" are announced.

The homotopic category of $K$, the category of (semi-) simplicial sets, is equivalent to the homotopy category of $W$, the category of spaces of homotopy type of a $C W$ complex [4, VII, 1], via a pair of adjoint functors. Moreover, in [8], the homotopic categories of $K$ and Cat, the category of small categories, are shown to be equivalent via the pair $N:$ Cat $\rightarrow K$ and $\Gamma: K \rightarrow$ Cat, where $N$ is the standard embedding nerve functor and $\Gamma$ is the category of simplices functor. As in the case for $K$ and $W$, one would like to replace $\Gamma$ by the left adjoint of nerve, categorical realization $c: K \rightarrow C$ at; however, $c$ is "wildly wrong" with respect to homotopy since it maps certain spheres to contractible categories. In this announcement, we give conditions for other "reasonable" functors from $K$ to $C$ at to be (weak) homotopy inverses for nerve. The functor $\Gamma: K \rightarrow$ Cat above and the functor $\Lambda: K \rightarrow$ Cat used in [11] are examples of such homotopy inverses.

We only consider functors from $K$ to $C$ at having right adjoints. Under very weak homotopy conditions, these right adjoints are homotopy equivalent to nerve

Received by the editors August 7, 1978.

AMS (MOS) subject classifications (1970). Primary 55D10, 18A40, 55D50; Secondary 55J10, $18 \mathrm{G} 30,55 \mathrm{~F} 35$.

Key words and phrases. Homotopy inverse for functor nerve, category of small categories, category of simplical sets, adjoint homotopy equivalence, divided functor, classifying space of small category, second barycentric subdivision functor. 
[10]. In addition, we give hypotheses under which a homotopy inverse and its right adjoint induce an adjoint equivalence between the homotopic categories of $K$ and Cat.

Particularly, the adjoint pair $c \cdot \mathrm{Sd}^{2}: K \rightarrow$ Cat and $\mathrm{Ex}^{2} \cdot N:$ Cat $\rightarrow K$ where $\mathrm{Sd}^{2}: K \rightarrow K$ is the second barycentric subdivision [5], gives such an adjoint equivalence. Thomason [18] used this adjunction to give a closed model structure (in the sense of Quillen [15]) for Cat. Furthermore, using special properties of $\mathrm{cSd}^{2}: K \rightarrow$ Cat, it follows that the geometric realization of any simplicial set is homeomorphic to the classifying space of a small category.

1. Preliminaries. The fundamental notions of homotopy theory in Cat and $K$, respectively, are discussed in $[\mathbf{1 0}, \mathrm{III}]$.

By $\Delta$ we denote the category of finite ordinals. According to [5] any pair of adjoint functors

$$
\Gamma_{\theta} \rightarrow S_{\theta}: \mathcal{C} \rightarrow K \equiv\left[\Delta^{\mathrm{op}}, \text { Ens }\right]
$$

is induced by $\theta=\Gamma_{\theta} \cdot \Delta: \Delta \rightarrow C$, where $C$ is a cocomplete category and $\Delta$ $\Delta \rightarrow K$ is the Yoneda embedding. In particular, $c \rightarrow N$ : Cat $\rightarrow K$ is induced by the canonical embedding $\iota: \Delta \rightarrow$ Cat. Furthermore, any natural transformation

$$
\eta: \theta \stackrel{\circ}{\rightarrow}: \Delta \rightarrow \text { Cat, }
$$

using adjoint functor theory $\left[9\right.$, IV], induces natural transformations $\eta_{2}: \Gamma_{\theta} \rightarrow$

$$
\eta_{2}: \Gamma_{\theta} \rightarrow c: K \rightarrow \text { Cat, } \eta^{\prime}: \Gamma_{N_{\theta}} \stackrel{\dot{\rightarrow}}{\rightarrow} \mathrm{Id}_{K}: K \rightarrow K .
$$

Moreover, the theory of coends [13, IX.6] guarantees that there exists a natural transformation

$$
\rho: \Gamma_{N \theta} \stackrel{\bullet}{\rightarrow} N \Gamma_{\theta}: K \rightarrow K
$$

making the following diagram commute

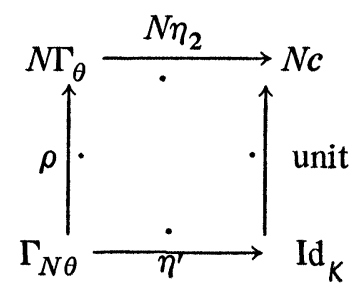

We introduce technical notions; these are necessary because $N$, as a right adjoint, does not generally preserve pushouts even up to homotopy. For the sake of brevity we restrict to one case and suppress the dual formulations.

1.3. An inclusion $i: \mathbf{A} \leftrightharpoons \mathbf{B}$ in Cat is coadmissible, if $b \in \mathbf{A}$ whenever $b$ is a morphism in $\mathbf{B}$ with codomain in A. If $R$ is a set of objects in the category $\mathbf{B}$, the admissible hull of $R$ in $\mathbf{B}$ is the full subcategory $\mathbf{Z}(R)$ of $\mathbf{B}$ generated by the morphisms in B with domain in $R$.

1.4. Let $f$ be a partial functor from $\mathbf{B}$ to $\mathbf{C}$, i.e. a functor in Cat defined 
as a subcategory $\operatorname{dom} f=\mathbf{A}$ of $\mathbf{B}$ with $\operatorname{cod} f=\mathbf{C}$. An object $p \in \mathbf{B}$ is a ramification object (with respect to $f$ ) if $p$ is the codomain of at least two different morphisms in $\mathrm{A}$ which are identified by $f$. We denote by $R_{f}$ the set of ramification objects in $B$ and by $W_{f}$ the full subcategory of $B$ generated by $A \cup Z\left(R_{f}\right)$.

1.5. Let $\theta: \Delta \rightarrow$ Cat be a functor. For any $n \geqslant 0$ we denote by $\dot{\theta}[n]$ the boundary of $\theta[n]$;i.e., the image of $\Gamma_{\theta} j_{n}: \Gamma_{\theta} \dot{\Delta}[n] \rightarrow \Gamma_{\theta} \Delta[n] \cong \theta[n]$ where $\dot{\Delta}[n]$ is the "simplicial" boundary of $\Delta[n]$ and $j_{n}: \dot{\Delta}[n] \hookrightarrow \Delta[n]$ denotes the inclusion. An object or morphism in $\theta[n]$ is interior if it does not belong to $\dot{\theta}[n]$.

1.6. A functor $\theta: \Delta \rightarrow$ Cat is divided, if

(i) for each face operator $\delta^{l}:[n-1] \hookrightarrow[n], 0 \leqslant i \leqslant n, 0 \leqslant n$, the inclusion $\theta \delta^{\imath}: \theta[n-1] \hookrightarrow \theta[n]$ is coadmissible;

(ii) for each degeneracy operator $\sigma^{t}:[n+1] \longrightarrow[n], 0 \leqslant i \leqslant n$, the epifunctor $\theta \sigma^{d}: \theta[n+1] \rightarrow \theta[n]$ has lifting with respect to codomain; i.e., given a morphism $c \in \theta[n]$ and an object $p \in \theta[n+1]$ with $\operatorname{cod} c=\left(\theta o^{l}\right) p$, there exists a (not necessarily unique) morphism $a \in \theta[n+1]$ such that $c=\left(\theta o^{d}\right) a$ and $p=\operatorname{cod} a$;

(iii) every morphism $b \in \theta[n]$ has a unique decomposition of the form $b=$ $(\theta \mu) a$ with $\mu$ face operator and $a$ interior.

1.7. Remark. If $\theta: \Delta \rightarrow$ Cat is a divided functor, then $\Gamma_{\theta}: K \rightarrow$ Cat preserves inclusions. Hence we can identify $\dot{\theta}[n]$ and $\Gamma_{\theta} \dot{\Delta}[n]$. In this situation, the functor $\Gamma_{\theta}$, applied to the terminal map $\dot{\Delta}[n] \rightarrow \Delta[0]$, yields a functor $\omega:$ $\dot{\theta}[n] \rightarrow \theta[0]$ which we shall consider as a partial functor from $\theta[n]$ to $\theta[0]$.

2. Statement of general results.

2.1. Theorem. Let $\theta: \Delta \rightarrow$ Cat be a divided functor. If for all $n \geqslant 0$, $\theta[n]$ is a coreflective subcategory of $\mathrm{W}_{\omega}$ (coreflective in the sense of [13, IV.3]), then

$$
\rho(X): \Gamma_{N \theta}(X) \rightarrow N \Gamma_{\theta}(X)
$$

is a weak homotopy equivalence (WHE) for all simplicial sets $X$.

The main tool used to prove this theorem is the following compatibility of nerve and pushout.

\subsection{THEOREM. Suppose $f$ is a partial functor from B to $\mathbf{C}$ such that}

(i) $\mathbf{A}=\operatorname{dom} f$ is a coadmissible subcategory of $\mathbf{B}$;

(ii) $f$ has lifting with respect to codomain;

(iii) $\mathbf{A}$ is a coreflective subcategory of $\mathrm{W}_{f}$.

Then the universal map $N \mathrm{~B} \amalg_{N \mathrm{~A}} N \mathrm{C} \rightarrow N\left(\mathrm{~B} \mathrm{U}_{\mathbf{A}} \mathrm{C}\right)$ is a WHE in $K$; i.e. nerve preserves pushouts of this form up to homotopy.

We assume that the conditions of 2.1 hold for the following corollaries. 
2.3. Corollary. Suppose that $\theta: \Delta \rightarrow$ Cat is as in (2.1) and that there exists a natural transformation (1.1) such that $\eta[k]: \theta[k] \rightarrow \iota[k]$ is a WHE for every $k \geqslant 0$. Then $\Gamma_{\theta}: K \rightarrow$ Cat is a weak homotopy inverse for $N:$ Cat $\rightarrow K$.

Namely $N \Gamma_{\theta}$ and $\mathrm{Id}_{K}$ are connected by

$$
N \Gamma_{\theta} \stackrel{\dot{\rho}}{\stackrel{\rho}{\rho}} \Gamma_{N \theta} \underset{\eta^{\prime}}{\stackrel{\dot{T}}{\longrightarrow}} \mathrm{Id}_{K}
$$

where $\rho X$ is a WHE by 2.1 , and from the subdivision theorem [8, Theorem 1], $\eta^{\prime} X$ is a WHE.

That the natural transformation

$$
\eta_{2} N: \Gamma_{\theta} N \stackrel{\rightarrow}{\rightarrow} N \cong \mathrm{Id}_{\mathrm{Cat}}
$$

yields WHE for every small category, follows from diagram (1.2), and (2.4).

2.5. Corollary. Suppose that $\theta: \Delta \rightarrow$ Cat as in (2.1) and that there exists a natural transformation (1.1) such that $\eta[k]: \theta[k] \rightarrow \iota[k]$ is a strong homotopy equivalence (SHE) for every $k \geqslant 0$. Then the adjunctions

induce WHE's

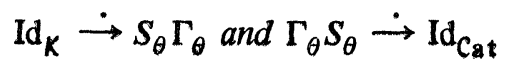

$$
X \rightarrow S_{\theta} \Gamma_{\theta} X \text { and } \Gamma_{\theta} S_{\theta} \mathbf{C} \rightarrow \mathbf{C}
$$

for all simplicial sets $\boldsymbol{X}$ and all small categories $\mathbf{C}$.

To see this one proves first using (1.2) and (2.1) that a map $f$ is a WHE in $K$ iff $\Gamma_{\theta} f$ is a WHE in Cat. The rest of the proof is precisely the same as the proof of Corollary 4.7 in [10].

3. The special case $\theta=c \Delta^{\prime}$. The functor $\Delta^{\prime}: \Delta \rightarrow K$ is defined in [5, 1.2]. Then $\theta=c \Delta^{\prime}: \Delta \rightarrow$ Cat is divided, but does not satisfy the extra hypoth. esis of 2.1. However, a weaker condition holds which yields the following theorem.

3.1. THEOREM: If $X$ is a regulated simplicial set $[12, \mathrm{III} .8]$, then $\rho(X)$ : $\Gamma_{N \theta}(X)=\operatorname{Sd}(X) \rightarrow N \Gamma_{\theta}(X)$ is a $W H E$.

We indicate another proof which gives a clearer interpretation of this result. It is based on:

3.2. Lemma. Let $X$ be a regulated simplicial set. Then $\Gamma_{\theta} X=\mathrm{cSd} X$ is a partially ordered set.

This may be shown by means of a straightforward computation and yields:

3.3. COROLLARY: If $X$ is a regulated simplicial set, then

$$
N \Gamma_{\theta}(X) \cong * X
$$

where * denotes the "star" functor in [12, III.9]. 
Finally combining results of Barratt (see [3] and [12]) and using a natural equivalence between $\mathrm{Sd}^{2}$ and $\mathrm{Sd} \cdot \mathrm{Sd}^{\mathrm{op}}$, we find:

3.4. THEOREM. $\mathrm{NcSd}^{2} X$ is the simplicial set of an ordered simplicial complex, for any simplicial set $X$. Moreover there is a homeomorphism $\left|\mathrm{NcSd}^{2} X\right| \rightarrow$ $|X|$. (Warning: This homeomorphism does not depend naturally on $X$.)

AcKnowledgements. The authors would like to thank Alex Heller, Saunders Mac Lane, John Moore, James Stasheff, Robert Thomason and Stephen Wilson for useful conversations, and North Carolina State University, Princeton University and the Universität Konstanz for partial support during this work.

\section{REFERENCES}

1. S. Eilen berg and J. A. Zilber, Semi-simplicial complexes and singular homology, Ann. of Math. 51 (1950), 499-513.

2. S. Eilenberg and S. Mac Lane, Acyclic models, Amer. J. Math. 75 (1953), 189-199.

3. R. Fritsch, Some remarks on $S$. Weingram: On the triangulation of a semi-simplicial complex, Illinois J. Math. 14 (1970), 529-535.

4. P. Gabriel and M. Zisman, Calculus of fractions and homotopy theory, Springer-Verlag, Berlin and New York, 1967.

5. D. M. Kan, On c.s.s. complexes, Amer. J. Math. 79 (1957), 449-476. $330-346$.

6. - Functions involving c.s.s. complexes, Trans. Amer. Math. Soc. 87 (1958),

7. - A combinational definition of homotopy groups, Ann. of Math. (2) 67 (1958), 282-312.

8. D. M. Latch, The uniqueness of homology for the category of small categories, J. Pure Appl. Algebra 9 (1977), 221-237.

9. - A fibred homotopy equivalence and homology theories for the category of small categories, J. Pure Appl. Algebra (to appear).

10. D. M. Latch, R. W. Thomason, and W. S. Wilson, Simplicial sets from categories, Math. Z. (to appear).

11. M. J. Lee, Homotopy for functors, Proc. Amer. Math. Soc. 36 (1972), 571-577; 42 (1974), 648-650.

12. A. T. Lundell and S. Weingram, The topology of $C W$ complexes, Van Nostrand, New York, 1969. 1971.

13. S. Mac Lane, Categories for the working mathematician, Springer-Verlag, New York,

14. J. Milnor, The geometric realization of a semi-simplicial complex, Ann. of Math. (2) (1957), 357-362.

15. D. G. Quillen, Homotopical algebra, Lecture Notes in Math., vol. 43, Springer-Verlag, New York, 1967.

16. - Higher alge braic K-theory. I, Higher $K$-theories, Lecture Notes in Math., vol. 341, Springer-Verlag, New York, 1973, pp. 85-147.

17. G. Segal, Classifying spaces and spectral sequences, Inst. Hautes Études Sci. Publ. Math. 34 (1968), 105-112.

18. R. W. Thomason, Cat as a closed model category (in preparation).

19. J. H. C. Whitehead, Combinatorial homotopy. I, Bull. Amer. Math. Soc. 55 (1949), 213-245.

FACHBEREICH MATHEMATIK, UNIVERSITÄT KONSTANZ, D 7750 KONSTANZ, WEST GERMANY

DEPARTMENT OF MATHEMATICS, NORTH CAROLINA STATE UNIVERSITY, RALEIGH, NORTH CAROLINA 27607 (Current address of Dana May Latch) 